\title{
Repercusiones de los sistemas digitales en la modalidad de teletrabajo en entidades públicas
}

Repercussions of digital systems in the telework modality in public entities

1 Francisco Xavier Rivera Lombeyda

https://orcid.org/0000-0001-9934-0029

Instituto Superior Tecnológico Vicente Rocafuerte, ISTVR,

Campus Vicente Rocafuerte Vélez y Lizardo García, Guayaquil, Ecuador

frivera@istvr.edu.ec

2 José Libinton Contreras Plua

https://orcid.org/ 0000-0003-2596-0967

Investigador Independiente

joselibintoncontrera@hotmail.com

Artículo de Investigación Científica y Tecnológica

Enviado: $11 / 11 / 2021$

Revisado: $26 / 11 / 2021$

Aceptado: 22/12/2021

Publicado:05/01/2022

DOI: https://doi.org/10.33262/concienciadigital.v5i1.1972

Rivera Lombeyda, F. X., \& Contreras Plua, J. L. (2022). Repercusiones de los sistemas digitales en la modalidad de teletrabajo en entidades públicas. ConcienciaDigital, 5(1), 88103. https://doi.org/10.33262/concienciadigital.v5i1.1972

CONCIENCIA DIGITAL, es una Revista Multidisciplinar, Trimestral, que se publicará en soporte electrónico tiene como misión contribuir a la formación de profesionales competentes con visión humanística y crítica que sean capaces de exponer sus resultados investigativos y científicos en la misma medida que se promueva mediante su intervención cambios positivos en la sociedad. https://concienciadigital.org

La revista es editada por la Editorial Ciencia Digital (Editorial de prestigio registrada en la Cámara Ecuatoriana de Libro con No de Afiliación 663) www.celibro.org.ec 


\section{Palabras} claves: repercusiones, sistemas digitales, modalidad de teletrabajo, servicio público.

\section{Keywords:}

(Repercussions, digital systems, teleworking modality, public service)
Resumen

Introducción. La pandemia de la COVID - 19 generó la imposición por decreto de la modalidad virtual de teletrabajo en las instituciones públicas. Objetivo. Revelar las repercusiones positivas y negativas del uso de los sistemas digitales en la modalidad de teletrabajo en entidades públicas. Metodología. Revisión bibliográfica. Resultados. Los empleados del sector público no estaban preparados para trabajar bajo la modalidad virtual, por lo que compraron recursos tecnológicos nuevos, incrementando sus gastos en el hogar y la incomodidad en el desarrollo de sus funciones, extendiéndose la jornada laboral diaria, a más de ocho horas, en donde se lidió también con problemas familiares. A pesar de ello, los empleadores, no realizaron la supervisión del estado de los recursos tecnológicos de los servidores públicos, ni participaron en sus gastos, porque no disponen de protocolos para su control. No obstante, el teletrabajo fue un aporte importante para garantizar la continuidad del trabajo, minimizando costos de desplazamiento y evitando la propagación del coronavirus. Conclusión. Se espera que se regule adecuadamente la modalidad de teletrabajo, para que los trabajadores puedan gozar de mejores condiciones de trabajo, ajustados a un horario definido, con equipos adecuados que aseguren mayor confortabilidad.

\section{Abstract}

Introduction. The COVID-19 pandemic generated the imposition by decree of the virtual modality of teleworking in public institutions. Objective. Reveal the positive and negative repercussions of the use of digital systems in the teleworking modality in public entities. Methodology. Bibliographic review.

Results. Public sector employees were not prepared to work under the virtual modality, so they bought new technological resources, increasing their expenses at home and the discomfort in the development of their functions, extending the daily workday to more than eight hours, where he also dealt with family problems. Despite this, the employers did not supervise the state of the technological resources of the public servants, nor did they participate in their expenses, because they do not have protocols for their control. However, teleworking was an important contribution to guarantee the continuity of work, minimizing travel costs and 
preventing the spread of the coronavirus. Conclusion. It is expected that the teleworking modality will be adequately regulated, so that workers can enjoy better working conditions, adjusted to a defined schedule, with adequate equipment that ensures greater comfort.

\section{Introducción}

La pandemia de la COVID-19 tomó por sorpresa al mundo entero, generando una crisis sanitaria, económica y social en todos los continentes, revolucionando la modalidad de trabajo que se mantenía en todos los sectores de la empresa pública y privada, no obstante, en el presente estudio solo se abordarán las entidades estatales, concentrándose en los empleados administrativos de 10 instituciones reconocidas de Guayaquil, para cumplir el objetivo de conocer las repercusiones de los sistemas digitales en la modalidad de teletrabajo que adoptaron estas instituciones del Estado, desde el mes de marzo del 2020.

La Organización Internacional del Trabajo (OIT), considera que el teletrabajo es una estrategia que pueda implementarse en todos los sectores económicos, gracias a la mayor conectividad que existe, debido a la evolución tecnológica acelerada de los últimos años, claro está, que se requiere de una masa laboral con mayor compromiso y preparación (Montero, et al., 2020). A ello se añade que, cuando la Organización Mundial de la Salud (OMS) declaró a la infección por coronavirus a nivel de pandemia, la mayoría de los países se acogió al teletrabajo como modalidad laboral, especialmente en las instituciones públicas, con la finalidad de preservar la salud de sus empleados y familiares, además de impedir la propagación del virus en la comunidad (Quispe y Quispe, 2021).

Si bien es cierto, la pandemia permitió la continuidad de los trabajos y el funcionamiento de los servicios en las entidades públicas, en todos los pueblos latinoamericanos (Camacho, 2021), sin embargo, ha generado algunas dificultades para estas instituciones. Según un estudio realizado en Bogotá, el teletrabajo ha generado mayor presión laboral en los empleados públicos, porque deben lidiar con los problemas del hogar y del trabajo al mismo tiempo (Triviño, 2020). Otro estudio realizado en Perú manifiesta que la separación del equipo de trabajo limita el desarrollo del conocimiento y de las experiencias por parte de los trabajadores, además que se expande el horario de trabajo, sin que sea reconocido por la empresa como horas extras (Barreda et al., 2021).

A pesar de las ventajas que ofrece el teletrabajo para las empresas públicas, porque reduce costos en infraestructura, espacio de oficina, expresos, movilizaciones de sus empleados, entre otros factores, no obstante, requiere de capacitación, reorganización de los trámites y procesos administrativos, además de infraestructuras tecnológicas capaces de dar 
soluciones en corto tiempo a las necesidades de empleadores y trabajadores. Sin embargo, el teletrabajo fue implementado sin siquiera un marco regulatorio, a partir de los decretos ejecutivos de confinamientos estipulados por los presidentes de las naciones latinoamericanas, donde se incluye a Ecuador, incrementando los costos en el personal administrativo, algunos de los cuales debieron invertir en oficinas en sus propios hogares, incrementándose el pago de servicios básicos, como es el caso de la energía eléctrica (Iturralde y Duque, 2021).

Esta situación la corroboró una investigación desarrollada en Guayaquil, que también evidenció percepciones de insatisfacción de los empleados, por lo que consideraron una modalidad injusta de trabajo, donde tienen que gastar más recursos económicos en el hogar, trabajando mayor cantidad de tiempo, por el mismo sueldo, con alta presión laboral e inclusive, con empleadores algo insatisfechos porque dudan de la honestidad de sus subordinados, estimando que no trabajan todo el tiempo de la jornada laboral, por dedicar tiempo a los miembros del hogar (Rugel y Romero, 2020).

Por este motivo, se seleccionó el tema de estudio, con el propósito de revelar las repercusiones positivas y negativas del uso de los sistemas digitales en la modalidad de teletrabajo en entidades públicas, que si bien es cierto, permiten la comunicación de todos los miembros de la empresa, aun encontrándose fuera de la oficina, sin embargo, pueden generar dificultades para los empleados y empleadores, los primeros porque se atienen a incrementos de costos en el desarrollo de sus labores, así como a jornadas extendidas de trabajo por el mismo sueldo y los segundos por la desconfianza que sienten por el uso de las horas laborables, por parte de sus subordinados, según lo que se ha evidenciado en los estudios efectuados sobre este tópico, hasta la fecha actual.

Es decir, que se justifica el desarrollo de la investigación, porque la modalidad virtual puede ser una de las herramientas más importantes que pueden utilizar las empresas, en el futuro inmediato, para lograr reducir costos y agilizar los procesos organizacionales, conforme a los requerimientos de la era moderna.

Pero, por otra parte, se necesitan normas, regulaciones, planificación, capacitación, entre otras actividades, para fortalecer la modalidad de teletrabajo (Gendler y Andonegui, 2021), en el Ecuador sobre todo en las instituciones públicas, donde se está tratando en la actualidad, el tema de la reducción de costos de la proforma presupuestaria y la disminución del gasto público (Moyón et al., 2020).

\section{Estado del arte}

Acerca de lo mencionado en los dos últimos párrafos, se ha tomado como referencia un artículo desarrollado en Perú, que indicó que el teletrabajo ha sido la única opción que tuvo el Estado para garantizar la continuidad de los servicios públicos, por lo tanto, a la 
preservación de la salud de los trabajadores, se suma la flexibilización del horario de labores y la disminución del tiempo de desplazamiento, como principales beneficios de esta modalidad de trabajo, aunque es necesario cubrir brechas tecnológicas y de conectividad, así como analizar los factores de riesgo ergonómico en el hogar y la convivencia de las relaciones familiares y laborales, afectada por el incremento de la presión laboral y la carga de trabajo (Quispe et al., 2021).

Otra investigación desarrollada en Colombia, en cambio, se centró en la obligatoriedad del a modalidad del teletrabajo en todo el territorio colombiano, a raíz de la llegada de la pandemia de la COVID-19, a pesar de las limitaciones tecnológicas existentes en algunos hogares de este país, ante una modalidad laboral que no ha sido regulada en los países latinoamericanos (Arango y Preciado, 2021).

A nivel nacional, el teletrabajo en el contexto de la pandemia de la COVID-19, ha desempeñado un rol muy gravitante en la continuidad de los servicios públicos y privados, para satisfacer las necesidades de la ciudadanía, evidenciándose las necesidades de las familias ecuatorianas por contar con recursos tecnológicos de alto nivel, para efectuar actividades eficientes bajo la modalidad laboral virtual, que además requiere la supervisión de condiciones seguras y confortables para los trabajadores, donde debe intervenir el compromiso de la dirección, por evitar eventos adversos de sus empleados que trabajan desde el hogar, que no solo significa evitar que se contagien de COVID-19, sino de protegerlos de riesgos físicos, eléctricos, mecánicos, ergonómicos, psicosociales, químicos, biológicos, entre otros (Lalama, 2021).

En el ámbito local, se abordó un tema que abarcó la modalidad de teletrabajo, bajo un enfoque normativo, donde pone de manifiesto que la emergencia sanitaria justificó el uso de esta modalidad laboral, pero ello no significa que, se inobserve los derechos de los trabajadores, quienes requieren condiciones seguras en el trabajo, porque se reconoce que la casa no fue planificada como una oficina, por lo que se puso énfasis en las normas y protocolos que deben plasmarse en la legislación nacional, para regular el teletrabajo y proteger la salud y los derechos laborales de los empleados del sector público y privado (Hernández, 2021).

Las investigaciones previas, manifiestan la importancia del teletrabajo para preservar la salud de los trabajadores, con relación a la minimización de la propagación comunitaria de la COVID-19. Sin embargo, exponen diferentes repercusiones en el ámbito familiar, de condiciones de trabajo, de conectividad y de relaciones laborales, propiamente dicho, donde inclusive puede existir una desconexión entre las partes empleadora y empleada, con riesgo de desprotección de los derechos de los trabajadores. 


\section{Teletrabajo}

El teletrabajo es según la literatura teórica, una modalidad basada en una nueva organización del trabajo, donde imperan las herramientas digitales representadas en las TIC, que no se desarrolla en la oficina ni en la infraestructura de la empresa, sino más bien en el hogar de los trabajadores (Giniger, 2020).

La medida de distanciamiento social, con el cometido de controlar la pandemia y minimizar su propagación comunitaria, ha generado que los gobiernos se refugien en el teletrabajo, como una modalidad para garantizar la continuidad de la atención a la ciudadanía, lo que puede tener una gran repercusión, tanto positiva como negativa en la comunidad de trabajadores y en las propias familias ecuatorianas (Perticará y Tejada, 2020).

Aunque el teletrabajo es una oportunidad para enfrentar la pandemia de la COVID-19 y mantener los puestos de trabajo, sobre todo de los grupos más vulnerables, porque las TIC han sido muy útiles para este propósito, no obstante, su implementación mediante decretos ejecutivos en las naciones latinoamericanas, ha sido incipiente, limitada y con muchas falencias, que carece de normas para proteger los derechos de los trabajadores y que lo expone a mayores riesgos laborales, debido a la falta de supervisión del domicilio de los empleados que realizan labores en sus domicilios, mediante la modalidad virtual (Camacho, 2021).

Bajo estas perspectivas, se realizará un estudio de campo sobre la modalidad virtual del teletrabajo, desde el punto de vista de los empleadores y empleados del sector administrativo de las instituciones públicas, previo señalamiento de los aspectos metodológicos a utilizar para el desarrollo de esta investigación.

\section{Metodología}

La metodología considerada para el presente artículo se fundamenta en la investigación descriptiva, la misma que permite obtener hallazgos inherentes a las causas y efectos de la problemática en estudio, con el propósito de revelar las repercusiones positivas y negativas del uso de los sistemas digitales en la modalidad de teletrabajo en entidades públicas (Rasinger, 2020).

También se ha considerado la aplicación de la investigación de campo, con un enfoque cuantitativo, logrando recolectar información correspondiente a las percepciones de insatisfacción de los empleados como repercusión de los sistemas digitales en la modalidad de teletrabajo en las entidades públicas (Rasinger, 2020).

Con relación a la población considerada en el estudio, para la obtención de la información se ha considerado el total de empleados del sector público que asciende a 41.767, mientras 
que, con relación a los representantes del empleador de las entidades públicas se obtiene un total de 4.177 jefes directos en las diferentes áreas (INEC, 2021). Por lo tanto, para efectos del cálculo de la muestra se consideran dos fórmulas, la primera aplicada para población infinita, ya que supera los 10.000 elementos participantes y la segunda fórmula se aplicada es para población finita, ya que los involucrados superan los 100 elementos.

Para el cálculo de los servidores públicos, se aplicó la siguiente formula (Valdivieso, 2017):

$$
\mathrm{n}=\frac{\mathrm{Z}^{2} \times \mathrm{p} \times \mathrm{q}}{\mathrm{d}^{2}}
$$

Simbología:

- $\mathrm{n}=$ Tamaño de muestra

- $\mathrm{p}=$ probabilidad de éxito $=0,5$

- $\mathrm{q}=$ probabilidad de fracaso $=0,5$

- $\quad \mathrm{d}^{2}=$ error de la medición (5\%).

- $\mathrm{Z}^{2}=$ Coeficiente de corrección del error $(1,96)$.

$\mathrm{n}=\frac{(1,96)^{2} \times(0,5) \times(0,5)}{(5 \%)^{2}}=384$ encuestas aplicadas a los servidores públicos

Mientras que, con relación a la población de los representantes del empleador de las entidades públicas, se presenta la siguiente fórmula:

$$
\mathrm{n}=\frac{\mathrm{N} \times \mathrm{Z}^{2} \times \mathrm{p} \times \mathrm{q}}{\mathrm{d}^{2} \times(\mathrm{N}-1)+\mathrm{Z}^{2} \times \mathrm{p} \times \mathrm{q}}
$$

Datos:

- Población $(\mathrm{N})=4.177$ representantes del empleador.

- $\quad$ Nivel de confianza $(Z)=1,96$

- $\quad$ Error $(d)=5 \%=0,05$

- Probabilidad de verdadero $(\mathrm{p})=50 \%=0,5$

- Probabilidad de falso $(q)=50 \%=0,5$

Desarrollando la ecuación:

$$
\mathrm{n}=\frac{(4.177) \times(1,96)^{2} \times(0,5) \times(0,5)}{(0,05)^{2} \times(4.177-1)+(1,96)^{2} \times(0,5) \times(0,5)}=352 \text { representantes del empleador }
$$


Concerniente a la técnica aplicada se ha seleccionado la encuesta definida como una herramienta que permite la recolección de información medible y cuantificable (Casas et al., 2018), mientras que, con relación al instrumento se consideró el cuestionario de preguntas cerradas, a través de la escala de Likert. Para el procesamiento de estos hallazgos se procedió a aplicar el cuestionario a los empleados y representantes de las entidades públicas, luego se tabuló los hallazgos y posteriormente se ha desarrollado el correspondiente análisis e interpretación referente a las repercusiones de los sistemas digitales en la modalidad de teletrabajo en entidades públicas.

\section{Resultados}

Los resultados fueron obtenidos mediante la aplicación de encuestas, tanto a trabajadores y empleadores de las entidades públicas, los cuales se describen en las tablas y figuras siguientes:

1) ¿Contaban los servidores públicos con equipos de computación adecuados, cuando les indicaron que debían trabajar la modalidad de teletrabajo?

\section{Tabla 1}

Disponibilidad de equipos de cómputos

\begin{tabular}{|c|c|c|c|c|c|}
\hline \multirow[b]{2}{*}{ Pregunta } & \multirow[b]{2}{*}{ Descripción } & \multicolumn{2}{|c|}{$\begin{array}{l}\text { Servidores } \\
\text { públicos }\end{array}$} & \multicolumn{2}{|c|}{$\begin{array}{c}\text { Representantes del } \\
\text { empleador }\end{array}$} \\
\hline & & Frecuencia & $\%$ & Frecuencia & $\%$ \\
\hline ¿Contaban los & Totalmente de & 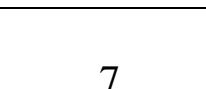 & $2 \%$ & 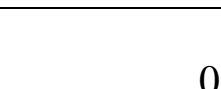 & $0 \%$ \\
\hline equipos de computación & De acuerdo & 97 & $25 \%$ & 0 & $0 \%$ \\
\hline $\begin{array}{l}\text { adecuados, cuando les } \\
\text { indicaron que debían }\end{array}$ & $\begin{array}{l}\text { Ni de acuerdo ni } \\
\text { en desacuerdo }\end{array}$ & 12 & $3 \%$ & 83 & $24 \%$ \\
\hline trabajar la modalidad de & En desacuerdo & 206 & $54 \%$ & 256 & $73 \%$ \\
\hline teletrabajo? & $\begin{array}{l}\text { Totalmente en } \\
\text { desacuerdo }\end{array}$ & 62 & $16 \%$ & 13 & $4 \%$ \\
\hline & Total & 384 & $100 \%$ & 352 & $100 \%$ \\
\hline
\end{tabular}

Fuente: Encuesta dirigida a los trabajadores de las entidades públicas.

Los hallazgos obtenidos con relación a la disponibilidad de equipos de cómputo adecuados para trabajar en la modalidad de teletrabajo por parte de los empleados de las entidades públicas describen que, en la mayoría de los casos los encuestados (54\%) están en desacuerdo, mientras que, el menos porcentaje (2\%), están totalmente de acuerdo. Estos resultados evidencian que los servidores públicos no disponían a los equipos para cumplir con la nueva modalidad digital de trabajo.

2) ¿Incrementaron los gastos en el hogar de los servidores públicos por el uso de equipos de computación para trabajar bajo la modalidad de teletrabajo? 
Tabla 2

Gastos adicionales en el hogar por teletrabajo

\begin{tabular}{|c|c|c|c|c|}
\hline & \multicolumn{2}{|c|}{ Servidores públicos } & \multicolumn{2}{|c|}{$\begin{array}{l}\text { Representantes } \\
\text { del empleador }\end{array}$} \\
\hline Descripción & Frecuencia & $\%$ & Frecuencia & $\%$ \\
\hline ¿Incrementaron los Totalmente $\mathrm{de}$ & & & & \\
\hline gastos en el hogar acuerdo & 31 & $8 \%$ & 0 & $0 \%$ \\
\hline de los servidores De acuerdo & 297 & $77 \%$ & 0 & $0 \%$ \\
\hline públicos por el uso $\mathrm{Ni}$ de acuerdo ni en & & & & \\
\hline de equipos de desacuerdo & 45 & $12 \%$ & 63 & $18 \%$ \\
\hline computación para En desacuerdo & 11 & $3 \%$ & 267 & $76 \%$ \\
\hline trabajar bajo la Totalmente & & & & \\
\hline modalidad de desacuerdo & 0 & $0 \%$ & 22 & $6 \%$ \\
\hline teletrabajo? & 384 & $100 \%$ & 352 & $100 \%$ \\
\hline
\end{tabular}

Fuente: Encuesta dirigida a los trabajadores de las entidades públicas.

Los hallazgos obtenidos con relación al incremento de los gastos en el hogar por el uso de equipos de computación para trabajar bajo la modalidad de teletrabajo describen que, en la mayoría de los casos los encuestados (77\%) están de acuerdo, mientras que, otro porcentaje (12\%) son indiferentes y un menor porcentaje (3\%) están en desacuerdo. Estos hallazgos evidencian que la población de servidores públicos incrementó sus gastos en el hogar debido al uso de los equipos de computación para trabajar bajo la modalidad de teletrabajo, como la luz, el internet e incluso en muchos casos la deuda de los equipos adquiridos.

3) ¿Son adecuadas las condiciones de trabajo para los servidores públicos en el hogar para laborar bajo la modalidad de teletrabajo?

Tabla 3

Condiciones adecuadas para el teletrabajo

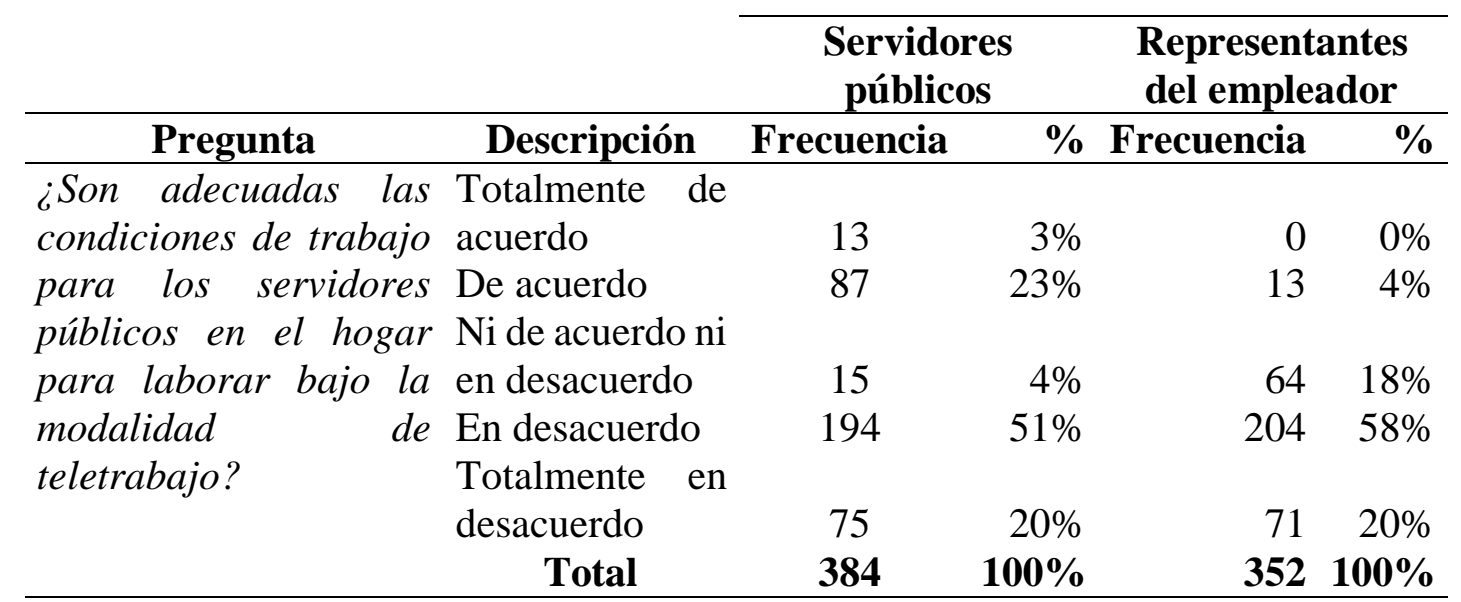

Fuente: Encuesta dirigida a los trabajadores de las entidades públicas. 
Los hallazgos obtenidos con relación a las condiciones adecuadas de trabajo en el hogar para laborar bajo la modalidad de teletrabajo describen que, en la mayoría de los casos los encuestados $(50 \%)$ están en desacuerdo, mientras que, un porcentaje significativo (23\%) está de acuerdo y un menor porcentaje (3\%) están totalmente de acuerdo. Estos hallazgos evidencian que la población de servidores públicos, en muchos casos no cuenta con las condiciones de trabajo adecuadas en el hogar, lo cual puede repercutir en el desarrollo de sus actividades y en el cumplimiento satisfactorio de su desempeño.

4) ¿Han tenido los empleados un horario de trabajo delimitado para laborar bajo la modalidad de teletrabajo?

Tabla 4

Delimitación de horario laboral

\begin{tabular}{|c|c|c|c|c|c|}
\hline & & $\begin{array}{l}\text { Servido } \\
\text { públic }\end{array}$ & & $\begin{array}{l}\text { Represent } \\
\text { del emple }\end{array}$ & $\begin{array}{l}\text { ntes } \\
\text { dor }\end{array}$ \\
\hline Pregunta & & & & Frecuenci & \\
\hline & Descripción & Frecuencia & $\%$ & $\mathbf{a}$ & $\%$ \\
\hline ¿Han tenido & Totalmente & & & & \\
\hline empleados un & acuerdo & 0 & $0 \%$ & 0 & $0 \%$ \\
\hline horario de trabajo & De acuerdo & 76 & $20 \%$ & 13 & $4 \%$ \\
\hline delimitado para & Ni de acuerdo ni en & & & & \\
\hline laborar bajo la & desacuerdo & 19 & $5 \%$ & 52 & $15 \%$ \\
\hline modalidad de & En desacuerdo & 193 & $50 \%$ & 204 & $58 \%$ \\
\hline teletrabajo? & Totalmente & & & & \\
\hline & desacuerdo & 96 & $25 \%$ & 83 & $\begin{array}{r}24 \% \\
100\end{array}$ \\
\hline & Total & 384 & $100 \%$ & 352 & $\%$ \\
\hline
\end{tabular}

Fuente: Encuesta dirigida a los trabajadores de las entidades públicas.

Los hallazgos obtenidos con relación a fijación de un horario de trabajo delimitado para laborar bajo la modalidad de teletrabajo describen que, en la mayoría de los casos los encuestados $(50 \%)$ están en desacuerdo, mientras que, un porcentaje significativo $(25 \%)$ está totalmente en desacuerdo y un menor porcentaje (5\%) son indiferentes al tema. Estos hallazgos evidencian que la población de servidores públicos no ha tenido un horario de trabajo delimitado para laborar bajo la modalidad de teletrabajo, lo cual impiden que puedan planificar el resto de sus actividades en el hogar y deban mantenerse conectados en todo momento dando lugar a la presión laboral.

5) ¿Considera que se la modalidad de teletrabajo constituye un aporte importante para el desarrollo de los sistemas digitales en el Ecuador, pero requiere regularse y potenciarse adecuadamente para garantizar su continuidad en el país? 


\section{Tabla 5}

Aportación del teletrabajo en los sistemas digitales

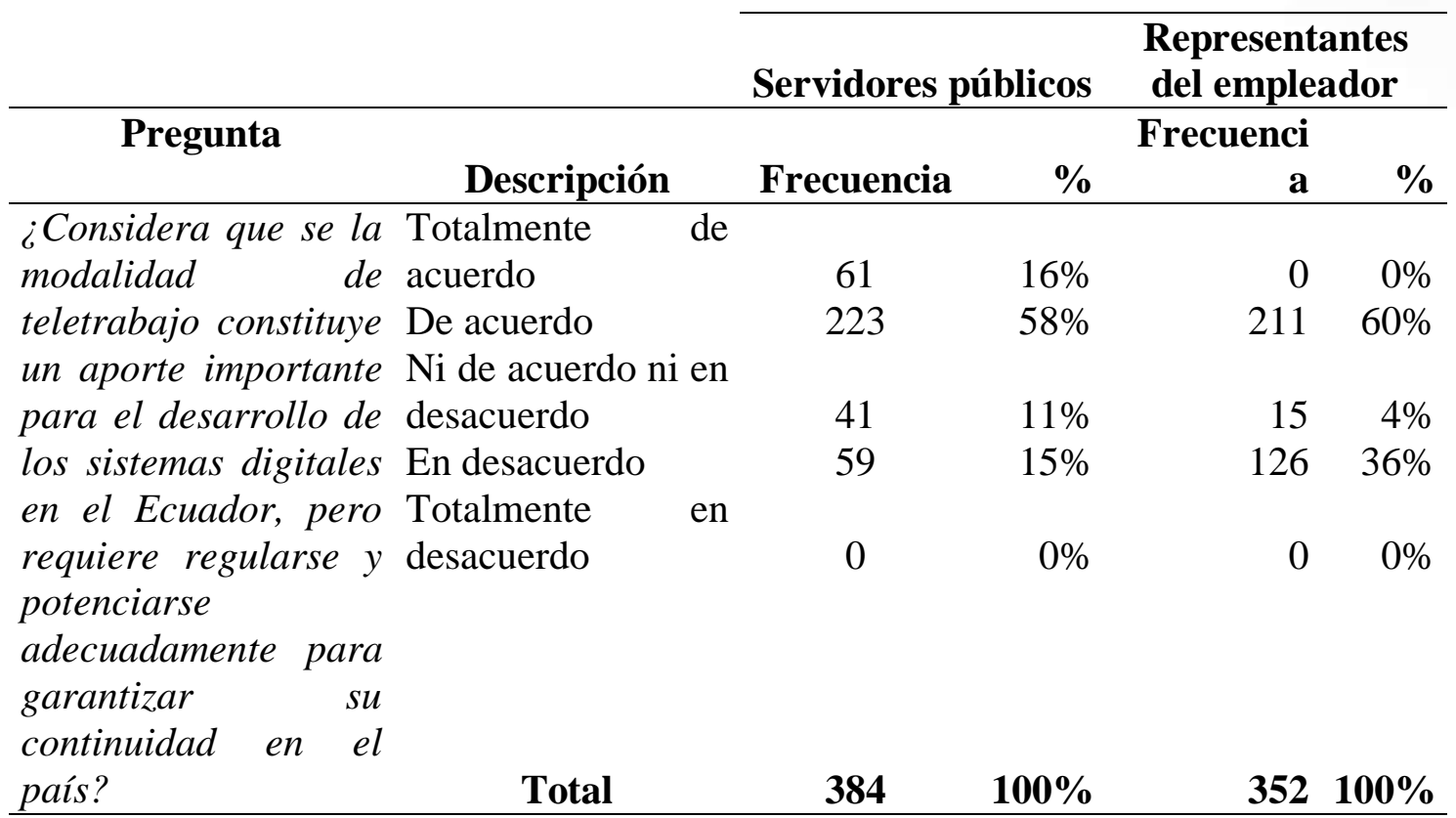

Fuente: Encuesta dirigida a los trabajadores de las entidades públicas.

Los hallazgos obtenidos con relación al aporte del teletrabajo para el desarrollo de los sistemas digitales en el Ecuador describen que, en la mayoría de los casos los encuestados (58\%) están de acuerdo, mientras que, un porcentaje menor (11\%) son indiferentes al tema. Estos hallazgos evidencian que la población de servidores públicos ha experimentado que la modalidad de teletrabajo es un aporte importante desarrollo de los sistemas digitales, sin embargo, se precisan normas, regulaciones, planificación, capacitación, entre otras actividades, para fortalecer la modalidad de teletrabajo y evitar la presión laboral.

\section{Discusión}

Los hallazgos obtenidos demostraron que más de la mitad de la muestra seleccionada de trabajadores (54\%), indicó que no estaba preparado para trabajar bajo la modalidad de trabajo y tuvo que comprar recursos tecnológicos, porque no contaba con equipos de computación con la capacidad suficiente para instalar sistemas y archivos pertenecientes a la empresa donde laboral, además que estos equipos también suelen ser utilizados por sus hijos y familiares, que estudian bajo la modalidad virtual o trabajan en otros establecimiento económico, por esta razón, más de las tres cuartas partes de los empleados (77\%), incrementó sus gastos en el hogar, esto sin menoscabo de que casi el $80 \%$ de trabajadores consideró que el teletrabajo fue un aporte importante para garantizar la continuidad de sus labores. 
Estos hallazgos son concordantes con los manifiestos de Quispe et al. (2021), que evidenciaron que el teletrabajo garantizó la continuidad de los servicios públicos, evitó la propagación del coronavirus en mayores proporciones a las ya obtenidas, flexibilizó el horario de labores y disminuyó el tiempo de desplazamiento, como ventajas principales. No obstante Arango y Preciado (2021), reconocieron como principales desventajas, limitaciones tecnológicas existentes en algunos hogares colombianos y la falta de regulación de esta modalidad, coincidiendo con los resultados de esta investigación.

A estos problemas se suman las condiciones inadecuadas de trabajo en el hogar, donde 7 de cada 10 trabajadores se quejaron porque no tiene confort, al laborar bajo la modalidad de teletrabajo, mientras que similar porcentaje de empleados no contó con un horario de trabajo definido, porque tuvieron que atender llamadas de sus jefes, en horas de la noche, fuera de la jornada laboral diaria normal. Además, 8 de cada 10 trabajadores manifestaron que, en sus domicilios, tuvieron que lidiar con problemas familiares, sobre todo en la educación virtual de sus hijos, donde asistieron a reuniones virtuales del establecimiento educativo de sus hijos, en horas de trabajo, además, que desarrollaron tareas en el hogar.

Estos hallazgos son comparables a los encontrados por el referente de Lalama (2021), quien evidenció limitada supervisión de la alta dirección de las empresas públicas y condiciones inseguras y poco confortables para los trabajadores, en la modalidad de teletrabajo. Mientras tanto Triviño (2020) manifestó que los empleados públicos, deben lidiar con los problemas del hogar y del trabajo al mismo tiempo, sin que haya podido controlarse este evento que es nocivo, tanto para la salud del empleado público, por el estrés que le genera, y, por el empleador que obtiene bajos desempeño en los procesos, coincidiendo con los resultados obtenidos en este estudio.

Los empleadores, en más del $80 \%$ de los casos, manifestaron en cambio, que ha sido imposible realizar la supervisión del estado de los recursos tecnológicos de sus trabajadores, porque no hay un método de control específico fijado para este particular, considerando que son equipos del empleado y no de la empresa, por ello, tampoco participaron en los gastos que realizó el empleado, ni participaron en la evaluación de riesgos laborales en el hogar del trabajador.

Estos resultados coincidieron con los obtenidos por Barreda et al. (2021), en el sentido de que, a pesar de la expansión del horario de trabajo en la modalidad virtual, este tiempo adicional, no fue reconocido por la empresa como horas extras, al igual que lo manifestado por la muestra seleccionada. Mientras que Iturralde y Duque (2021), se centraron en la limitada supervisión de los recursos tecnológicos y no reconocimiento de los gastos realizados por los empleados, por parte de la empresa pública, sucesos que también se reflejaron en los hallazgos de este artículo. 
Además, debido a que el $80 \%$ de las empresas no ha regulado la actividad de teletrabajo, a través de protocolos documentados, tampoco ha regulado un horario de trabajo, por lo que, la población mayoritaria estimó que es necesario regular esta modalidad de trabajo, para garantizar la continuidad del empleo en el país.

Estos resultados también coincidieron con los mencionados por Hernández (2021), quien se refirió a la falta de regulación de la modalidad de teletrabajo, situación que está vulnerando los derechos de los trabajadores, motivo por el cual, es necesario regula la modalidad virtual del trabajo y dotarla de un horario definido, así como de mayor confortabilidad para los empleados, en procura de garantizar la continuidad de la jornada laboral mientras dure la pandemia, conservando la calidad del trabajo y respetando los derechos laborales.

\section{Conclusiones}

- Se pudo identificar que los empleados del sector público no estaban preparados para trabajar bajo la modalidad virtual, por ello, tuvieron que comprar recursos tecnológicos nuevos, para cumplir con sus tareas laborales en el hogar, incrementando sus gastos en el hogar y trabajando con incomodidad, debido a las condiciones inadecuadas de trabajo en el hogar, donde no se definió la extensión de la jornada laboral diaria y se tuvo que lidiar al mismo tiempo, con los problemas familiares, propios del hogar. A pesar de ello, el teletrabajo fue un aporte importante para garantizar la continuidad de sus labores, minimizando costos de desplazamiento y evitando la propagación del coronavirus entre los empleados.

- Asimismo, se identificó que los empleadores, no realizaron la supervisión del estado de los recursos tecnológicos de sus trabajadores, por la falta de un método de control específico fijado para este particular, por ello, no participaron en los gastos que realizó el empleado, ni participaron en la evaluación de riesgos laborales en el hogar del trabajador, porque esta modalidad virtual laboral, no se reguló en protocolos organizacionales, coincidiendo con los empleados, en que esta forma de trabajo, está garantizando la continuidad de la jornada laboral mientras dure la pandemia.

- A pesar de ello, se espera que se regule adecuadamente la modalidad virtual en el país, para que los trabajadores puedan gozar de mejores condiciones de trabajo, ajustados a un horario definido, con equipos adecuados que pertenezcan al empleador y a condiciones de mayor confortabilidad, para potenciar el desempeño laboral y la salud de los trabajadores. 


\section{Referencias bibliográficas}

Arango, V., \& Preciado, D. (2021). Teletrabajo y trabajo en casa: diferencias y posibilidades en el marco de la pandemia por COVID-19. Medellín: Universidad EAFIT.

Barreda, S., Escalante, A., \& Picoy, A. (2021). Influencia de la percepción de las condiciones del trabajo no presencial en la satisfacción laboral de los profesores de posgrado durante la pandemia por la COVID-19. Lima: ESAN Graduate School Of Business.

Camacho, J. (2021). El teletrabajo, la utilidad digital por la pandemia del COVID-19. Revista latinoamericana de derecho social, 2(32), 15.

Casas, J., Labrador, J., \& Donado, J. (2018). La encuesta como técnica de investigación. Elaboración de cuestionarios y tratamiento estadístico de los datos (I). Atención primaria: Elsevier, 31(8), 15.

Gendler, M., \& Andonegui, F. (2021). El COVID-19 y las regulaciones digitales en Argentina: modificaciones y desarrollos en una pandemia inédita. CONICET, 12(22), 28.

Giniger, N. (2020). Teletrabajo. Modalidad de trabajo en pandemia. Observatorio Latinoamericano y El Caribe, 4(1), 17.

Herbas, B., \& Rocha, E. (2018). Metodología científica para la realización de investigaciones de mercado e investigaciones sociales cuantitativas. Perspectivas no. 42 Cochabamba nov., http://www.scielo.org.bo/scielo.php?script=sci_arttext\&pid=S1994$37332018000200006 \& \operatorname{lng}=\mathrm{es} \& \mathrm{nrm}=$ iso.

Hernández, N. (2021). Normativa del Teletrabajo en el Ecuador en la Actual Crisis Sanitaria del COVID 19. Guayaquil: Universidad Católica de Santiago de Guayaquil.

INEC. (2021). Encuesta Nacional de Empleo, Desempleo y Subempleo. Ecuador: https://www.ecuadorencifras.gob.ec/empleo-ene-2021/.

Iturralde, C., \& Duque, L. (2021). Precarización del teletrabajo en Ecuador en contexto de COVID-19: variables de análisis desde el enfoque marxista. Revista Chakiñan de Ciencias Sociales y Humanidades, 2(14).

Lalama, O. (2021). Análisis de las condiciones en que se desarrolla el teletrabajo en el contexto de la pandemia Covid-19, caso de estudio de trabajadores de una empresa privada del cantón Guayaquil. Guayaquil: Universidad de Guayaquil. 
Montero, B., Vasconcelos, K., \& Arias, G. (2020). Teletrabajo: fortaleciendo el trabajo en tiempos de pandemia por COVID-19. Revista de Comunicación y Salud, 10(2), 17.

Moyón, L., Robalino, G., \& Gallegos, D. (2020). Análisis comparativo del Presupuesto General del Estado y su influencia en la política fiscal en el Ecuador, periodo 2019-2020. QUIPUKAMAYOC, 28(56), 10.

Perticará, M., \& Tejada, M. (2020). Sobre vulnerabilidad y teletrabajo durante la pandemia. Observatorio Económico, 1(1), 10.

Quispe, A., \& Quispe, G. (2021). Teletrabajo en las entidades públicas, una necesidad y prioridad en la actualidad. Ciencia Latina, 5(3), 21.

Quispe, E., Reaño, F., Gambetta, I., \& Córdoba, S. (2021). Actitudes y percepciones de líderes de empresas de Lima Metropolitana durante la etapa inicial de la pandemia COVID-19. Surco, Perú: Pontifica Universidad Católica del Perú.

Rasinger, S. (2020). La investigación cuantitativa en Linguística. Argentina: AKAL. Obtenido https://books.google.es/books?hl=es\&lr=\&id=0h4EEAAAQBAJ\&oi=fnd\&pg=P $\mathrm{A} 8 \& \mathrm{dq}=$ investigaci\%C3\%B3n+cuantitativa\&ots $=8 \mathrm{~L} 0 \mathrm{hZKz} \_\mathrm{Sa} \& \operatorname{sig}=4 \mathrm{Kr} 1 \mathrm{Az}-$

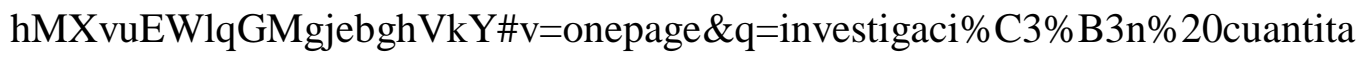
tiva\& $\mathrm{f}=$ true

Rugel, L., \& Romero, R. (2020). Las percepciones de los trabajadores frente al teletrabajo durante la pandemia Covid-19, caso de estudio realizado a los residentes de una urbanización del Cantón Daule. Guayaquil: Universidad Católica Santiago de Guayaquil.

Triviño, J. (2020). Influencia del cambio de modalidad laboral (teletrabajo) y el aislamiento en el bienestar y calidad de vida durante la emergencia por COVID19. Bogotá: Institución Universitaria Politécnica Grancolombiana.

Valdivieso, C. (2017). Determinación del tamaño muestral mediante el uso de árboles de decisión. Investigación y Desarrollo, 11(2), 15. 
El artículo que se publica es de exclusiva responsabilidad de los autores y no necesariamente reflejan el pensamiento de la Revista Conciencia Digital.

\section{Ligital}

El artículo queda en propiedad de la revista y, por tanto, su publicación parcial y/o total en otro medio tiene que ser autorizado por el director de la Revista Conciencia Digital.
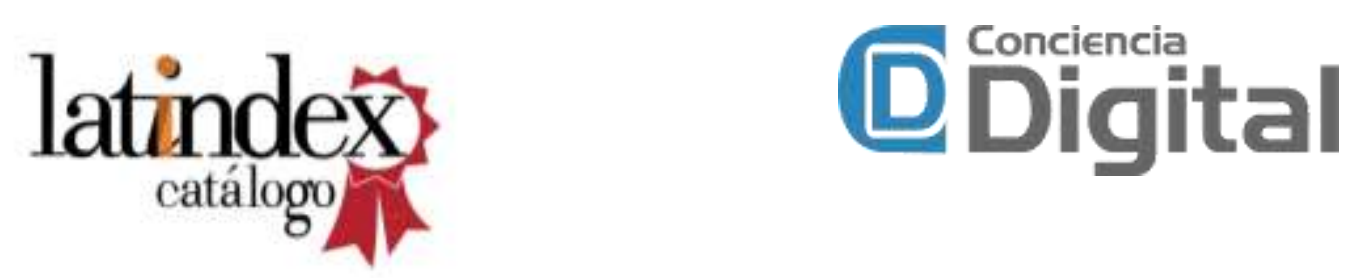

Indexaciones

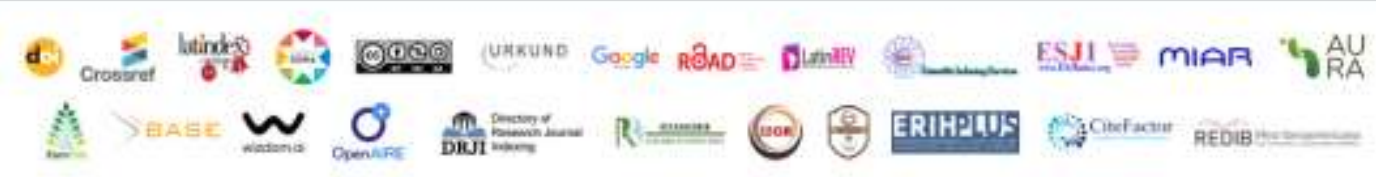

